\title{
Image Restoration using 3-Dimensional Discrete Cosine Transform
}

\author{
D. Ramesh Varma \\ M.Tech (DECS) \\ Department of ECE \\ Vishnu Institute of Technology
}

\author{
G. Prasanna Kumar \\ Assistant Professor \\ Department of ECE \\ Vishnu institute of technology
}

\author{
P. S. N. Murthy \\ Professor \\ Department of ECE \\ Vishnu institute of technology
}

\begin{abstract}
Image restoration is one of the major issues in the field of image processing. Generally, images are corrupted due to the ageing effect. The previous techniques are based on only single domain i.e.., either local domain or non-local domain. This paper present a new technique called "Image restoration using 3-dimensional discrete cosine transform" which comprises of two domains. Initially, smoothing of image is performed with the help of horizontal and vertical difference operators in the local domain and then synthesizes the textures with the help of discrete cosine transform in non-local domain. A split bregman iterative algorithm is developed to make these two domains more tractable and robust. In this paper, the problem of removing text in an image and filling the corrupted regions is dealt with the help of proposed technique. The proposed method achieves significant performance improvements over the existing state-of-art schemes.
\end{abstract}

\section{Keywords}

Discrete cosine transform, ageing effect, Split-bregman algorithm

\section{INTRODUCTION}

In image science, image restoration is one of the key issues which have to be sorted in an efficient way. The images have to be restored in different perspectives i.e. removing of blurness in the image which is occurred due to camera shake or atmospheric instability and due to the noise present in the system and due to ageing effect within the pixels of an image. The restored image can be obtained by reconstructing the original image from its degraded version. Image prior knowledge plays an important role in restoring an image.

$$
l=G m+n .
$$

Where $m$ represents the original image and $l$ represents the degraded image. $n$ represents the noise and $G$ is a mask i.e. the matrix whose diagonal elements are either o or 1 which corresponds to killing or keeping pixels which becomes problem of image inpainting [1], [2].

As everyone knows that the prior knowledge of an image plays a crucial role in restoring an image, there is a minimization problem which is given as;

$$
\min _{m} 0.5\|G m-l\|_{2}^{2}+\alpha H(m) \ldots .
$$

Where first term in (2) represents precision of data and regularization term denoting prior knowledge of image is represented by $\mathrm{H}(\mathrm{m})$. Equation (2) is nothing but a regularized framework which is derived from Bayesian inference which can be explained in the following sections. Many algorithms have been developed to solve minimization problem but the key is that they should have prior knowledge about the figure which is core work for designing regularization term. Many regularization terms are built on the assumption that smoothness of an image is within local region except at the edges. This type of regularization terms usually smear out the details at the edges. The proposed method has two domains in which both smoothness and edge enhancement is done accurately. Firstly, we have smoothed the image with the help of filters in local domain and synthesized the textures with the help of discrete cosine transform in non-local domain. The text removal in the image is done in these domains because of its consistency.

This paper is organised as follows. Section I intricate Introduction. Section II elaborates restoration of image within the local regions i.e. maintaining local consistency of an image. Section III shows restoration of image within nonlocal regions i.e. synthesizing the textures and maintaining global consistency of an image. Section IV elaborates establishment of hybrid domain. Section V elaborates algorithm, Flowchart, sequential diagram of the proposed method. Section VI elaborates experimental results.

\section{LOCAL RESTORATION OF AN IMAGE}

Local restoration of an image deals with smoothing an image within the local regions [See Fig.1 Blue colour region]. Here, we prefer natural images whose responses for high pass filters are small.

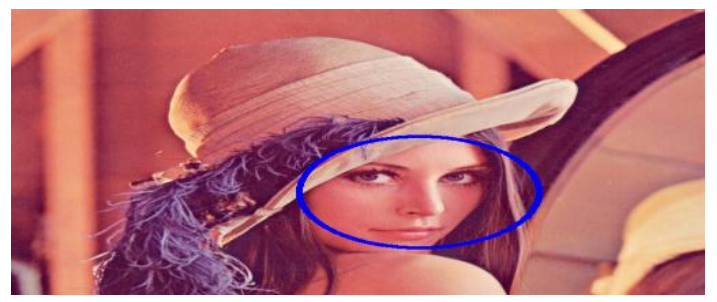

Fig.1. Smoothing an image within local region (blue colour region)

Smoothing an image within the local region is done by filtering operations by taking two filter masks i.e. horizontal filter mask and vertical filter mask which can be expressed as $F_{H}=[1-1]$ and $F_{V}=[1-1]^{T}$ whose statistics are given by the generalized Gaussian distribution [3] which can be expressed as

$$
\rho(m \mid E, s, \alpha)=\frac{1}{|E|^{\frac{1}{2}}} g_{s, \alpha}\left(m^{T} E^{-1} m\right) \ldots .
$$

Where $\mathrm{E}$ is the $p \times p$ symmetric matrix and $\mathrm{g}($.$) is the density$ operator which is given as 


$$
g_{s, \alpha}(l)=\frac{\alpha \gamma\left(\frac{p}{2}\right)}{\pi^{\frac{p}{2}} \gamma\left(\frac{p}{2 \alpha}\right) 2^{\frac{p}{2 \alpha}}} \frac{1}{s^{\frac{p}{2}}} \exp \left(-\frac{l^{\alpha}}{2 s^{\alpha}}\right) \ldots
$$

Here $\alpha$ is the scale parameter and $s$ is the shape parameter. More discussion about the $s$ value and $\alpha$ value can be found in [3].

By placing $\alpha=0.5$ in (3) to get laplacian distribution to model marginal statistics which is expressed as,

$$
\mathcal{R}_{L}(m)=|| F(m)\left\|_{1}=|| F_{h}(m)\right\|_{1}+\| F_{v}(m)||_{1} \ldots
$$

The above equation clearly shows that the characterization of smoothness in an image is achieved along with convex optimization. From equation (5) it is clear that it has low complexity.

\section{GLOBAL RESTORATION OF AN IMAGE}

Global restoration of an image deals with synthesizing the textures [4], [5], [6] and maintaining nonlocal consistency of an image. Here, the self-similarity between the images is considered for synthesizing the textures [See Fig.2 Yellow colour regions].

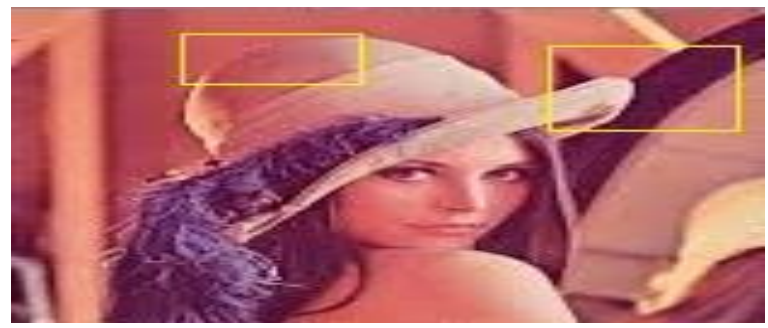

Fig.2. Texture synthesis within nonlocal region (yellow colour region)

The previous methods in the literatures perform restoration of an image in a weighted manner using nonlocal weighted graph. Recently quite impressive results have obtained by BM3D [7] and JSM [8] methods. The proposed method comprises of 3D discrete cosine transform which is having very good advantage of high energy compaction i.e.., most of the energy is concentrated in fewer coefficients [See Fig.3]. For texture synthesis, the neighbouring pixels should be highly correlated. In block based DCT, the DC coefficient describe the intensity of an image and $\mathrm{AC}$ coefficients describe direction information of an image.

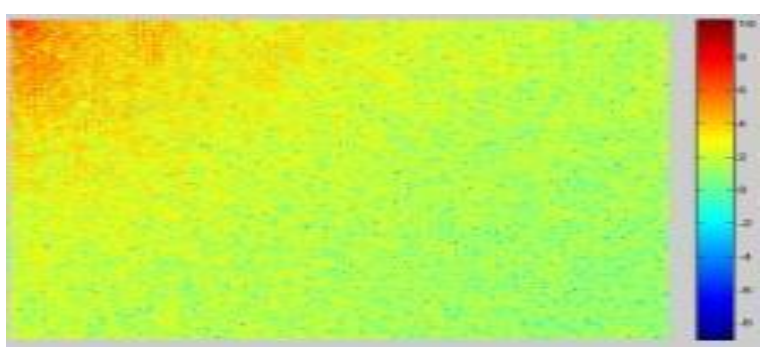

Fig.3. Energy compaction of an image

The texture synthesis is done in following steps.
1. Choose an image in which texture synthesis has to be done.

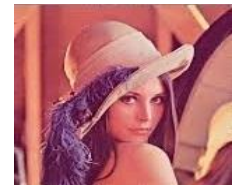

2. Choose the regions of textures synthesis. Those regions are represented by yellow colour regions

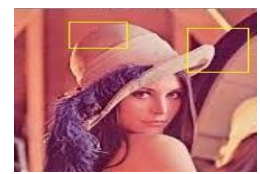

3. Choose another region \{Red colour region\} around texture region \{Yellow colour region\} to find selfsimilarity between the pixels of an image.

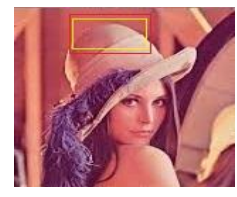

4. Divide the texture region into ' $n$ ' number of blocks

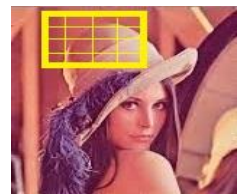

5. Calculate Euclidean distance between red colour block and each block in yellow colour \{texture region\}.

6. The blocks which are having low Euclidean distance is taken as self-similar images.

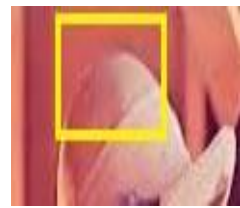

7. Arrange them in a 3D stack form.

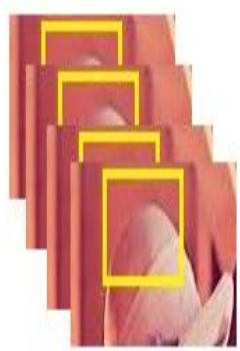

8. Transform coefficients are obtained by using 3D transform.

9. Applying inverse transform leads to generation of estimates for each block in 3D array. The final image estimate is achieved by averaging the block-wise estimates.

$$
\mathcal{R}_{G}(m)=\|3 D\|_{1}=\sum_{i=1}^{n}\left\|\mathrm{~T}^{3 D}\right\|_{1} \ldots(6)
$$


Above equation shows the mathematical representation of synthesizing the textures in an image.

\section{ESTABLISHMENT OF HYBRID DOMAIN}

The hybrid domain is formed by combining the two domains i.e. the local domain in which smoothing of image is done and the nonlocal domain in which texture synthesis is done. This domain will richly portray the smoothness in the image and synthesizes the textures which will increase the quality of reconstruction. A split-bregman iterative algorithm [9] is used to make this domain more robust and tractable which helps in solving inverse problem and optimization problem.

$$
\mathcal{R}_{H}(a)=\partial .\|F(m)\|_{1}+\wp .\|3 D\|_{1} \ldots \ldots \ldots(7)
$$

\section{ESTABLISHMENT OF SYSTEMATIC ALGORITHM FOR ITERATIONS}

For effectively restoration the image, the noise should be zero i.e. $G m-l=0$. But, due to noise present in the channel, it is not possible. So, the noise should be kept very much near to the value of zero. In the proposed method, the split bregman iterative algorithm is used to remove the text in an image which is nothing but a process of Image inpainting. In this algorithm the concept of bregman distance is used which can be explained in [9].

Constrained optimization via bregman iteration [9], [10] can be done by

$$
\min E(m) \text { such that } G m=l
$$

The constrained problem is converted into unconstrained minimization problem by introducing penalty function

$$
\min E(m)+\frac{\lambda}{2}\|G m-l\|_{2}^{2}
$$

For smaller values of $\lambda$, the penalty function does not accurately enforce the constraint. This problem can be solved by performing iterations.

$$
\begin{gathered}
m^{i+1}=\operatorname{minE}(a)+\frac{\lambda}{2}\left\|G m-l^{i}\right\|_{2}^{2} \ldots \\
l^{i+1}=l^{i}+l+G m^{i} \ldots \ldots(10) \\
\underset{\substack{L t \\
i \rightarrow \infty}}{G m^{i}=l}
\end{gathered}
$$

In this paper, the implementation of proposed algorithm is done using the below equation.

$$
\begin{gathered}
\left(m^{i+1}, c^{i+1}\right)= \\
\min _{m, c}|c|+Y(m)+\frac{\lambda}{2} \| c^{i}-Q m-\left.l^{i}\right|_{2} ^{2} \ldots \\
l^{i+1}=l^{i}+\left[Q\left(m^{i+1}\right)-c^{i+1}\right] \ldots . .
\end{gathered}
$$

\section{Generalized split bregman algorithm}

$$
\text { While }|| m^{i}-\left.m^{i-1}\right|_{2}>\text { tol }
$$

For $\mathrm{n}=1$ to $\mathrm{N}$

$$
\begin{aligned}
& m^{i+1}=\min _{m} Y(m)+\frac{\lambda}{2}|| c^{i}-Q m-\left.l^{i}\right|_{2} ^{2} \ldots \\
& c^{i+1}=\min _{c}|c|+\frac{\lambda}{2}|| c-Q\left(m^{i+1}\right)-\left.l^{i}\right|_{2} ^{2} \ldots .
\end{aligned}
$$

End

$$
l^{i+1}=l^{i}+\left[Q\left(m^{i+1}\right)-c^{i+1}\right] \ldots
$$

End

By incorporating the above proposed algorithm into the regularized framework, the equation can be written as

$$
\left.\operatorname{argmin}_{m} \frac{1}{2}\|G m-l\|\right|_{2} ^{2}+\partial . \mathcal{R}_{L}(m)+\wp \cdot \mathcal{R}_{G}(m) \ldots
$$

In the above equation $v$ and $\mathfrak{f}$ represents the constraint variables. First term of (16) represents the observation constraint, second and third terms represent the local and nonlocal preliminaries (priors) of an image.

Firstly, state

$$
\begin{array}{r}
Y(m)=\frac{1}{2}\|G m-l\|_{2}^{2} \ldots \ldots \ldots(17) \\
z(t)=z(Q m)=\partial \cdot \mathcal{R}_{L}(m)+\wp \cdot \mathcal{R}_{G}(m) \ldots .
\end{array}
$$

By inducing SBI algorithm into framework then

$$
\begin{aligned}
m^{i+1} & =\min _{m} Y(m)+\frac{\lambda}{2}\left\|c^{i}-Q m-l^{i}\right\|_{2}^{2} \\
& =\frac{1}{2}\|G m-l\|_{2}^{2}+\frac{\lambda}{2}\left\|\left[\begin{array}{c}
w^{i} \\
x^{i}
\end{array}\right]-\left[\begin{array}{l}
I \\
I
\end{array}\right] m-\left[\begin{array}{c}
a^{i} \\
b^{i}
\end{array}\right]\right\|_{2}^{2} \ldots
\end{aligned}
$$

Where $c^{i}=\left[\begin{array}{l}a^{i} \\ b^{i}\end{array}\right] \in R^{2 N}, a^{i}, b^{i} \in R^{N}$

By using variable splitting technique, we have

$$
\begin{aligned}
m^{i+1}= & \min _{m} \frac{1}{2}|| G m-\left.l\left\|\left.\right|_{2} ^{2}+\frac{\lambda}{2}|| w^{i}-m-a^{i}\right\|\right|_{2} ^{2} \\
& +\frac{\lambda}{2}\left\|x^{i}-m-b^{i}\right\|_{2}^{2} \ldots \ldots .(20)
\end{aligned}
$$

Next, by using SBI algorithm

$$
c^{i+1}=\left[\begin{array}{c}
w^{i+1} \\
x^{i+1}
\end{array}\right]=\min _{w, x}\left\{\begin{array}{c}
\partial . \mathcal{R}_{L}(m)+\wp \cdot \mathcal{R}_{G}(m) \\
+\frac{\lambda}{2}\left\|w-m^{i+1}-a^{i}\right\|_{2}^{2} \\
+\frac{\lambda}{2}\left\|x-m^{i+1}-b^{i}\right\|_{2}^{2}
\end{array}\right\} \ldots
$$

Clearly, the minimization w.r.t $\mathrm{w}, \mathrm{x}$ are decoupled leading to

$$
\begin{gathered}
w^{i+1}=\min _{w} \partial . \mathcal{R}_{L}(m)+\frac{\lambda}{2}|| w-m^{i+1}-\left.a^{i}\right|_{2} ^{2} \ldots . \\
x^{i+1}=\min _{x} \wp . \mathcal{R}_{G}(m)+\frac{\lambda}{2}|| x-m^{i+1}-\left.b^{i}\right|_{2} ^{2} \ldots \ldots
\end{gathered}
$$

According to SBI algorithm, the update of $\mathrm{l}_{\mathrm{i}}$ is given by

$$
l^{i+1}=\left[\begin{array}{l}
a^{i+1} \\
b^{i+1}
\end{array}\right]=\left[\begin{array}{l}
a^{i} \\
b^{i}
\end{array}\right]-\left(\left[\begin{array}{l}
I \\
I
\end{array}\right] m^{i+1}-\left[\begin{array}{c}
w^{i+1} \\
x^{i+1}
\end{array}\right]\right) \ldots .
$$

Solving three subproblems such as $m, x, w$ will solve the minimization problem (16). The three subproblems are explained in [8]. The complete algorithm for minimizing (16) is given as

Input: Image $l$ and linear matrix $\mathrm{G}$

Initialization: $\mathrm{i}=0, \mathrm{~m}^{(0)}=l, \mathrm{a}^{(0)}=\mathrm{b}^{(0)}=\mathrm{w}^{(0)}=\mathrm{x}^{(0)}=0, \partial, \wp, \lambda$

Repeat:

$$
m^{i+1}=\min _{m} \frac{1}{2}|| G m-\left.l\right|_{2} ^{2}
$$




$$
\begin{aligned}
& +\frac{\lambda}{2}\left\|w^{i}-m-a^{i}\right\|_{2}^{2}+\frac{\lambda}{2}\left\|x^{i}-m-b^{i}\right\|_{2}^{2} \\
& \mathrm{~s}^{i}=m^{i+1}-a^{i} ; \gamma=\frac{\partial}{\lambda} ; \\
& w^{i+1}=\operatorname{prox}\left(\mathcal{R}_{L}\right)\left(s^{i}\right) ; \\
& h^{i}=m^{i+1}-b^{i} ; \alpha=\frac{\wp}{\lambda} ; \\
& x^{i+1}=\operatorname{prox}\left(\mathcal{R}_{G}\right)\left(h^{i}\right) ; \\
& \quad a^{i+1}=a^{i}-\left(m^{i+1}-w^{i+1}\right) ; \\
& b^{i+1}=b^{i}-\left(m^{i+1}-x^{i+1}\right)
\end{aligned}
$$

Until: Condition of stopping criteria

Output: Restored image $m$

\section{FLOW CHART, ALGORITHM OF PROPOSED ALGORITHM}

\section{Algorithm of Proposed Algorithm}

1. Take the damaged image.

2. Resize the image $[256,256]$

3. Construct the hybrid domain in which smoothing an image and texture synthesis is done for an image which is corrupted by the text on it. The removal of text in an image and filling the regions of damaged pixels is done by following steps

Input: Image $l$, matrix $\mathrm{G}$

Initialization: $\mathrm{i}=0, \mathrm{~m}^{(0)}=l$,

$\mathrm{a}^{(0)}=\mathrm{b}^{(0)}=\mathrm{x}^{(0)}=\mathrm{w}^{(0)}=0, \partial, \wp, \lambda$

Repeat:

Compute $\mathrm{m}^{(\mathrm{i}+1)}[9,10,11,12]$

$m=\frac{1}{\tilde{\lambda}}\left(I-\frac{1}{1+\tilde{\lambda}} G^{T} G\right)$. B

Compute $\mathrm{w}^{(\mathrm{i}+1)}$ by Fast iterative shrinkage algorithm $[13,14]$

$l_{i+1}=m_{i}+\left[\frac{t_{i}-1}{t_{i}+1}\right] \cdot\left(m_{i}-m_{i-1}\right)$

Compute $\mathrm{x}^{(\mathrm{i}+1)}$ by soft threshold[ 9]

$x=\Psi_{G}\left(\Delta\left(3 D_{r}, \sqrt{2 \beta}\right)\right.$

$a^{i+1}=a^{i}-\left(m^{i+1}-w^{i+1}\right)$

$$
b^{i+1}=b^{i}-\left(m^{i+1}-x^{i+1}\right)
$$

Until: Condition is satisfied

Output: Final restored image.

4. For enhancing the edges, apply un-sharp filter while preserving the smoothness of an image.

\section{Flow chart of Proposed Algorithm}

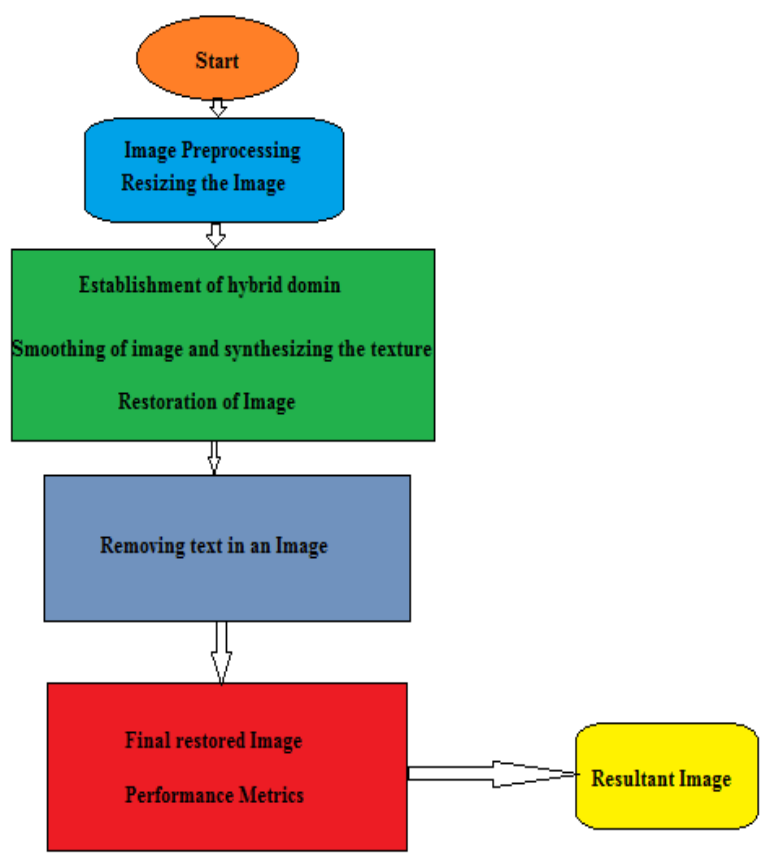

Fig.4. Flowchart of Proposed Composite Algorithm

\section{EXPERIMENTAL RESULTS}

1. Image Inpainting

Here, image inpainting [15], [16], [17] is taken as a case of text removal. The main theme of text removal is to obtain the original image by recovering the damaged regions which are degraded due to the pixels of the text. In this paper, different aspects like object recognition, text removal and filling the holes in an image are dealt. Image inpainting is done in following steps i.e.

- $\quad$ First read the damaged image and the mask to fill it.

- Clear damaged area in the original damaged area with the help of killing or keeping the pixels with 0 or 1 .

- Based on neighbourhoods, perform isotropic diffusion or dilation or erosion inside the damaged area or arrange a mask which is containing 0 's or 1 's. This concept is clearly explained in [18-]

$$
\left(G^{T} G+\tilde{\lambda}\right)^{-1}=\frac{1}{\tilde{\lambda}}\left(I-\frac{1}{1+\tilde{\lambda}} G^{T} G\right)
$$

The matrix in this equation is a diagonal with elements either

$$
\frac{1}{\tilde{\lambda}} \text { or } \frac{1}{1+\tilde{\lambda}}
$$

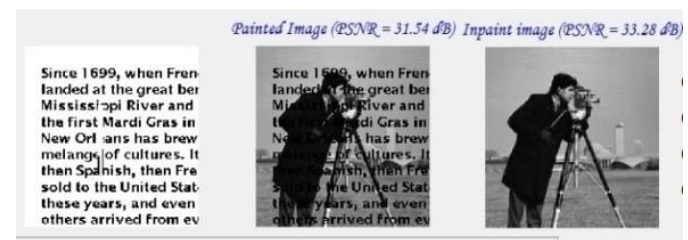

Fig.5. Experimental results for text removal in an image 

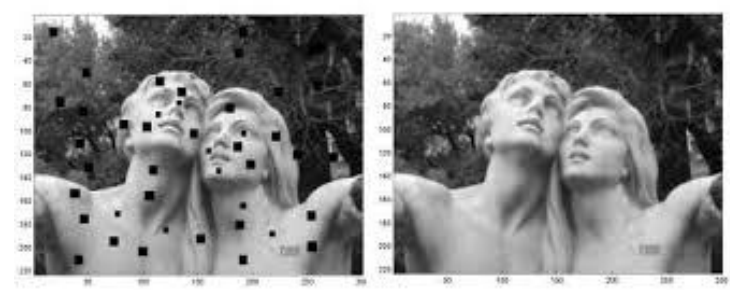

Fig.7. Pictorial representation of object recognition smoothness in an image. The explanation about unsharp filter is explained in [22].
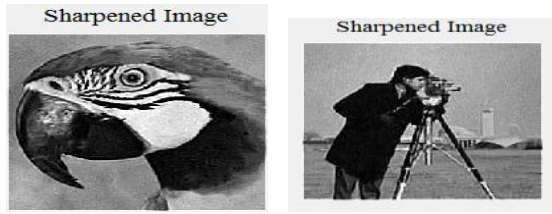

Fig.8. Sharpened images

\section{Image sharpening}

Image sharpening is done to enhance the edges of the image. In our proposed method we have used unsharp filter which is used to enhance the edges in an image while preserving the

Table.1. Performance Metrics of Previous Techniques

\begin{tabular}{|c|c|c|c|c|c|}
\hline Method & $\begin{array}{l}\text { Process of } \\
\text { approach }\end{array}$ & Image & $\begin{array}{l}\text { Initial } \\
\text { PSNR } \\
\text { of an } \\
\text { image }\end{array}$ & $\begin{array}{c}\text { Image } \\
\text { Inpainting } \\
\text { PSNR(db) }\end{array}$ & $\begin{array}{l}\text { Sharpened } \\
\text { image } \\
\text { PSNR }(\mathrm{db})\end{array}$ \\
\hline \multirow[t]{3}{*}{ SA-DCT } & \multirow[t]{3}{*}{ Hard-thresholding } & Cameraman & 23.41 & 30.25 & 30.92 \\
\hline & & Parrot & 23.03 & 31.25 & 31.36 \\
\hline & & Peppers & 23.95 & 36.25 & 37.06 \\
\hline \multirow[t]{3}{*}{ SALSA } & \multirow[t]{3}{*}{ Total Variation } & Cameraman & 23.41 & 31.68 & 32.96 \\
\hline & & Parrot & 23.03 & 32.02 & 33.25 \\
\hline & & Pepper & 23.95 & 26.53 & 30.56 \\
\hline \multirow[t]{3}{*}{ JSM } & \multirow{3}{*}{$\begin{array}{l}\text { Combination of } \\
\text { cosine and } \\
\text { wavelet transform }\end{array}$} & Cameraman & 23.41 & 32.98 & 34.55 \\
\hline & & Parrot & 23.03 & 33.04 & 35.20 \\
\hline & & Peppers & 23.95 & 38.40 & 39.22 \\
\hline
\end{tabular}


Table.2. Performance Metrics of Proposed technique

\begin{tabular}{|l|c|c|c|c|c|}
\hline \multirow{4}{*}{ HDA } & \multirow{2}{*}{$\begin{array}{c}\text { Filters and Cosine } \\
\text { Transform }\end{array}$} & Cameraman & 23.41 & 33.28 & 35.03 \\
\cline { 3 - 6 } & & Parrot & 23.03 & 33.32 & 35.69 \\
\cline { 3 - 6 } & & & & \\
& & Peppers & 23.95 & 38.71 & 40.59 \\
\hline
\end{tabular}

In this paper, the analysis is performed with the help of different combinations of 3D transform which are tabulated below [Table.3]. These transforms are used just for estimating the behaviour of various transform coeeficients for image inpainting.

Table.3. Performance metrics using different combinations of 3D transform

\begin{tabular}{|c|c|c|c|c|}
\hline \multirow{3}{*}{ 2D-DCT+1D-db8 } & Cameraman & 23.41 & 32.27 & 32.96 \\
\hline & Parrot & 23.03 & 32.14 & 32.85 \\
\hline & Peppers & 23.95 & 36.43 & 36.92 \\
\hline \multirow{3}{*}{ 2D-DCT+1D-1.5bior } & Cameraman & 23.41 & 31.43 & 31.76 \\
\hline & Parrot & 23.03 & 31.76 & 31.98 \\
\hline & Peppers & 23.95 & 35.67 & 36.06 \\
\hline
\end{tabular}

\section{CONCLUSION}

In this paper, a new algorithm for image restoration using hybrid domain is proposed, which characterizes the smoothness and synthesizes the textures at a time. In this paper, the texture synthesis is done by using 3 dimensional discrete cosine transform which gave good results when compared to the current state-of-art schemes. Experimental results on Image inpainting i.e.., removing text in the image and filling the damaged regions have shown that the proposed method achieves good performance metrics. In this paper, we have made an analysis by using different combinations of 3D transform like daubechies, wavelets, Biorthogonal and cosine transforms which are used to analyze the response of various transforms for the textures synthesis. Also, we have tabulated the results which shows that the proposed method give better PSNR values and better visualization when compared to the weighted methods like SALSA and SA-DCT. This method can be applied to various applications like Image deblurring and Image denoising.

\section{ACKNOWLEDGEMENT}

We are delighted to have great opportunity to thank the authors of [3-12] for kindly providing the necessary information. We feel pleasure to thank my professors, teaching and non-teaching staff of my department and my friends for their notable and valuable suggestions which leads to successful completion of our work.

\section{REFERENCES}

[1] "Image Inpainting-Automatic Detection and Removal of Text From Images" by Uday Modha and Preeti Dave

[2] "Region filling and Object Removal by Exemplar-Based Image Inpainting" by A. Criminisi, P. P'erez and K. Toyama

[3] "Parametric generalized gaussian density estimation" by M.K.Varanasi and B.Aazhang.

[4] "Compression artifact reduction by overlapped-block transform coefficient estimation with block similarity" by X.Zhang, R.Xiong, S.Ma, X.Fan and W.Gao.

[5] "Point wise shape-adaptive DCT for high quality denoising and deblocking of grayscale and color images" by V.Katkovnik, A.Foi and K.Egiazarian.

[6] "Texture synthesis by non-parametric sampling" by T.K.Leung and A.Efros.

[7] "Image restoration by sparse 3D transform-domain collaborative filtering" by K.Dabov, A.Foi and V.katkovnik.

[8] "Image restoration using Joint statistical modelling in a space-transform domain" by Jian Zhang, Debin Zhao, Wen GAO, Siwei Ma and Ruiqin Xiong.

[9] "The split Bergman iterative algorithm for L1 regularized problems" by T.Goldstein and S.osher.

[10] "Split Bregman methods and frame based image restoration," by J. F. Cai, S. Osher, and Z. W. Shen 
[11] "Non-local discrete regularization on weighted graphs" by A.Elmaotaz, S.Bougleux and O.Lezoray.

[12] R.Fergus and D.Krishnan "Fast image deconvolution using hyper laplacian priors".

[13] "Fast gradient-based algorithms for constrained total variation image denoising and deblurring problems," by A. Beck and M. Teboulle.

[14] "A new TwIST: Two-step iterative shrinkage/thresholding algorithms for image restoration" by J.Bioucas-Dias and M.Figueiredo.

[15] "3D data denoising and inpainting with the fast curvelet transform” by A. Woiselle, J. L. Starck, and M. J. Fadili.

[16] J. Yang, Y. Wang, H. Wang, K. Hua, W. Wang, and J. Shen. "Automatic objects removal for scene completion".
[17] P. P. A. Criminisi and K. Toyama. "Object Removal by Exemplar-Based Inpainting.

[18] T. Shih and R. Chang, "Digital Inpainting-Survey and Multilayer Image Inpainting Algorithms".

[19] K. Jain, and B. Yu, "Automatic Text Location in Images and Video Frames"

[20] "Simultaneous cartoon and texture image inpainting using morphological component analysis" by M.Elad, L.Starck, P.Querre and D.L.Donoho.

[21] "Patch-based Texture Synthesis for Image Inpainting" by Tao Zhou, Johnson.

[22] Image sharpening using un-sharp filters 